\title{
Errata: "Universality in Orthogonal and Symplectic Invariant Matrix Models with Quartic Potential”*
}

\section{ALEXANDRE STOJANOVIC}

Institut Mathématique de Jussieu, Physique mathématique et Géométrie, Université Paris 7 , case 7012, 2 Place Jussieu, 75251 Paris Cedex 05, France.e-mail: stojanov@math.jusssieu.fr

Abstract. The errata concern mainly the last computations for the universality of the local statistics of eigenvalues at the edge of the spectrum in parts (iii) of Theorems 2.3 and 2.4.

\section{Corrections}

- Page 343, (1.20): there is a factor $1 / 2$ in front of the right-hand side.

- Page 346, (2.10): It can be simplified in

$$
g_{j k}=a_{j k}-\sum_{\ell=k-d}^{2 n-1} \sum_{m=2 n-d}^{2 n-1} s_{j m} t_{m \ell} a_{\ell k} .
$$

- Page 347, before Theorem 2.3: The definition of $b(x, y)$ must be

$$
b_{\beta}(x, y)=\frac{1}{2} \operatorname{Ai}(x)\left(c_{\beta}-\int_{y}^{+\infty} \operatorname{Ai}(t) \mathrm{d} t\right), \quad \text { with } c_{\beta}= \begin{cases}1, & \text { if } \beta=1, \\ 0, & \text { if } \beta=4 .\end{cases}
$$

The precedent definition corresponds to $c_{\beta}=1 / 2$, what is wrong.

- Page 347, Theorem 2.3, part (ii): $-s^{\prime}(x-y)$ must be replaced by $s^{\prime}(x-y)$ in the expression of the matrix kernel $\tau_{1}(x, y)$.

- Page 348, Theorem 2.3, part (iii):

$$
\left(Z_{j}-\frac{w}{c_{j} n^{2 / 3}}, Z_{j}+\frac{v}{c_{j} n^{2 / 3}}\right)
$$

must be replaced by

$$
\left(Z_{j}-\frac{w_{j}}{c_{j} n^{2 / 3}}, Z_{j}+\frac{v_{j}}{c_{j} n^{2 / 3}}\right)
$$

with $w_{2}=w, v_{2}=v, w_{1}=-v$, and $v_{1}=-w$, because $\operatorname{sign}\left(c_{j}\right)=(-1)^{j}$.

$\theta_{1}(x, y)$ is now only defined by the first expression, in which we replace $b(x, y)$ by $b_{1}(x, y)$, the limit is now taken independently of the parity of $n$ and the result holds for $j=1$ and $j=2$.

\footnotetext{
^ Math. Phys. Anal. Geom. 3(4) (2000), 339-373.
} 
Moreover, we have to multiply by $(-1)^{j}$ the matrix kernel $\theta_{1}\left(x_{p}, x_{q}\right)$ in the expression of the limiting scaled correlation functions.

- Page 349, Theorem 2.4, part (ii): $-s^{\prime}(2(x-y))$ must be replaced by $s^{\prime}(2(x-y))$ in the expression of the matrix kernel $\tau_{4}(x, y)$.

- Page 349, Theorem 2.4, part (iii): We modify the expression of the interval as in the case $\beta=1$.

Replace $b(x, y)$ by $b_{4}(x, y)$ in the expression of $\theta_{4}(x, y)$ and multiply it by $1 / 2$.

- Page 350, Theorem 2.4, part (iii): We have to multiply by $(-1)^{j}$ the matrix kernel $\theta_{4}\left(x_{p}, x_{q}\right)$ in the expression of the limiting scaled correlation functions.

- Page 363: In fact, we have

$$
\lim _{n \rightarrow \infty} \frac{1}{c_{j} n^{2 / 3}} K_{n}\left(Z_{j}+\frac{x}{c_{j} n^{2 / 3}}, Z_{j}+\frac{y}{c_{j} n^{2 / 3}}\right)=(-1)^{j} a(x, y) .
$$

- Page 367, last line: We have $(-1)^{\sigma_{0}+j}$ at the numerator of the fraction.

- Page 369 , last line: replace $(-1)^{n+p}$ by $(-1)^{2 n+p}$.

- Page 371, bottom: In fact $c^{\prime}$ has the same value as $c$ if $n$ is odd.

\section{Explanations}

We give explanations for the mistakes about the end of the computations for the universality of the local statistics of eigenvalues at the edge of the spectrum. The mistakes in the proof of the computations of the terms depending on $H_{n}$ and $G_{n}$ have a common origin: if $\|\cdot\|_{\infty}:=\|\cdot\|_{L^{\infty}\left(\left(Z_{j}-\delta, Z_{j}+\delta\right)^{2}\right)}$, then we have said that

$$
\begin{aligned}
& \left\|\psi_{n+p} \otimes\left(\varepsilon \star \psi_{n+q}\right)-\psi_{n+q} \otimes\left(\varepsilon \star \psi_{n+p}\right)\right\|_{\infty}=\mathrm{O}\left(n^{-1 / 2}\right), \\
& \left\|\left(\varepsilon \star \psi_{n+p}\right) \otimes\left(\varepsilon \star \psi_{n+q}\right)-\left(\varepsilon \star \psi_{n+q}\right) \otimes\left(\varepsilon \star \psi_{n+p}\right)\right\|_{\infty}=\mathrm{O}\left(n^{-7 / 6}\right),
\end{aligned}
$$

which is wrong. There are some contributions at the level of constants of integration, which make wrong the arguments based on the antisymmetry. Thus, the estimates, pages 363-364, for $\varepsilon_{\mu} \star G_{n}, \varepsilon_{\lambda} \star \varepsilon_{\mu} \star G_{n}$ and for the part called $\beta_{n}$ in the expression of $H_{n}$ are false. Since now, there are contributions of $\varepsilon_{\mu} \star G_{n}$, $\varepsilon_{\lambda} \star \varepsilon_{\mu} \star G_{n}, \varepsilon_{\mu} \star \beta_{n}$ and $\varepsilon_{\lambda} \star \varepsilon_{\mu} \star \beta_{n}$ and the method of computations is completely different at this level. Now, we need equivalents of the coefficients $g_{j k}$ and not only estimates, what requires more computations. The details are given in the revision www. physik. uni-bielefeld.de/bibos/preprints, 02-07-098, BiBoS, Bielefeld, May 2002 of the preprint [13]. The method consists in doing what we have done on pages 367-368, for the computations of the contribution of the term $\alpha_{n}(\lambda)$. First, we have to give explicitly the values of the coefficients $g_{j k}$ in function of the coefficients $a_{j k}, a_{j k}^{\prime}$ and $c_{j k}$. Secondly, we have to give the equivalents of these coefficients $g_{j k}$; these coefficients are rational functions in terms of the coefficients $a_{j k}, a_{j k}^{\prime}$ and $c_{j k}$. The denominators are factors from the expression of det $D$; equivalents are given on pages $370-372$. We just have to compute the 
equivalents of the numerators to get the results. We give the results for the new versions of Lemmas 4.3 and 4.4:

- For $\beta=1, n$ even, we have

$$
\begin{aligned}
& g_{n-3, n-2}=\frac{n \sqrt{-t}}{4}(\sqrt{1+u}-\sqrt{1-u})+\mathrm{O}\left(n^{2 / 3}\right), \\
& g_{n-1, n-2}=\frac{n \sqrt{-t}}{2} \sqrt{1-u}+\mathrm{O}\left(n^{2 / 3}\right) .
\end{aligned}
$$

- For $\beta=1, n$ odd, we have

$$
\begin{aligned}
& g_{n-2, n-3}=\frac{n}{4} \sqrt{-t}(-\sqrt{1+u}+\sqrt{1-u})+\mathrm{O}\left(n^{2 / 3}\right), \\
& g_{n-1, n-2}=\mathrm{O}\left(n^{2 / 3}\right) .
\end{aligned}
$$

- For $\beta=4$, we have

$$
\begin{aligned}
g_{2 n, 2 n+1}= & \frac{n}{4} \sqrt{-2 t}(\sqrt{1+\sqrt{2} u}+\sqrt{1-\sqrt{2} u}) \times \\
& \times\left(\frac{\sqrt{1+\sqrt{2} u}-(-1)^{n}\left(1-2 u^{2}\right)^{1 / 4}}{\sqrt{1+\sqrt{2} u}+(-1)^{n}\left(1-2 u^{2}\right)^{1 / 4}}\right)+\mathrm{O}\left(n^{2 / 3}\right), \\
g_{2 n+1,2 n+2}= & \frac{n}{4} \sqrt{-2 t}[(\sqrt{1+\sqrt{2} u}+\sqrt{1-\sqrt{2} u})- \\
& -(\sqrt{1+\sqrt{2} u}-\sqrt{1-\sqrt{2} u}) \times \\
& \left.\times\left(\frac{\sqrt{1+\sqrt{2} u}-(-1)^{n}\left(1-2 u^{2}\right)^{1 / 4}}{\sqrt{1+\sqrt{2} u}+(-1)^{n}\left(1-2 u^{2}\right)^{1 / 4}}\right)\right]+ \\
+ & \mathrm{O}\left(n^{2 / 3}\right) .
\end{aligned}
$$

